\title{
Atrial Fibrillation One of Hidden Causes of Subclinical Stroke: Case-Control Study
}

\author{
Haider Shaheed Mohammed \\ Lecturer, Department of Internal Medicine, College of Medicine, University of Thi-Qar, Thi-Qar, Iraq
}

\begin{abstract}
Background: Silent cerebral infarction is frequently encountered on various imaging modalities of brain in individuals with atrial fibrillation, its exact relation with atrial fibrillation is still debated.

Objectives: The study was carried out to estimate the link between silent cerebral infarcts with atrial fibrillation, both paroxysmal and persistent types.

Materials and Method. A random sample of 150 were included in the study, 100 with atrial fibrillation 50 case with paroxysmal, 50 case with persistent type, and 50 control, for both cases and control a questionnaire was constructed, physical examination, neurological examination, and magnetic resonance imaging were done.

Results: Mean age was 64.6667. Of the total sample there was 96 males and 54 females. 89 individual of the total sample had silent cerebral lesion while 61 had no lesion. Of those who had lesions, 38 individuals had paroxysmal atrial fibrillation, 41 had persistent atrial fibrillation, and only 10 individuals belong to control group. There was high statistically significant difference between group of cases with atrial fibrillation and group of control in sinus rhythm where $\mathrm{p}$ value is 0.0001 .
\end{abstract}

Conclusion: Both paroxysmal and persistent atrial fibrillation is associated with heightened hazard of silent cerebral infarctions.

Keywords: Paroxysmal atrial fibrillation, persistent atrial fibrillation, silent cerebral infarctions.

\section{Introduction}

Atrial fibrillation is customarily happened arrhythmic heart disorders in clinical ground [1]. It is think about to be the most continual arrhythmia in older age individuals [2]. Worldwide its prevalence and incidence is get higher ${ }^{[3]}$, with almost one percent prevalence over the world ${ }^{[4]}$. Atrial fibrillation is a hazard for recruitment of cardiac failure, health facility consumption, uprising morbidity burden, and fatality [1]."Paroxysmal atrial fibrillation is defined as an episode

\section{Corresponding Author"}

\section{Haider Shaheed Mohammed}

(M.B.Ch.B.C.A.B.M.S), Lecturer, Department of Internal Medicine, College of Medicine, University of Thi-Qar, Thi-Qar, Iraq e-mail: hayder.sh@utq.edu.iq of atrial fibrillation that terminate spontaneously or with intervention in less than seven days"[13]."Persistent atrial fibrillation is defined as atrial fibrillation that fails to terminate within seven days"[13]. Worldwide stroke listed as the second most frequent cause for fatality and the third habitual cause for disability ${ }^{[5]}$. Stroke is remarkably catastrophic danger in Asia and its danger of death in Asia is more than western nations ${ }^{[6]}$. Atrial fibrillation is a noteworthy predisposing condition for ischemic stroke by mechanism of thromboembolization and the stroke that is attended by atrial fibrillation is more dangerous, with worse sequelae, greater disablement, higher fatality and massive infarct size than those without atrial fibrillation ${ }^{[7]}$. Atrial fibrillation heighten the hazard of embolic stroke by five times and the hazard is alternating from one to fifteen percent every year dependent on attendance of comorbid disorders ${ }^{[8]}$. Atrial fibrillation is not only give rise to symptomatic 
stroke but also asymptomatic one that is brain ischemia in individuals with atrial fibrillation wandering from clinically asymptomatic to catastrophic ${ }^{[9]}$. Silent cerebral infarction is referred to existing of brain infarction without parallel complaints [10]. Silent cerebral infarction happened in both individuals with atrial fibrillation and those without, but nearly most of studies stated that it is happened with raised occurrence in clients with atrial fibrillation ${ }^{[4]}$. The prevalence in general public fluctuates correlating to the imaging method implemented, the prevalence of silent cerebral infarction discovered by magnetic resonant imaging in most estimations falling in range of eight to twentyeight percent, while is approximately forty percent in patients with atrial fibrillation ${ }^{[9]}$. Silent cerebral infarction is well-recognized to advance to symptomatic stroke [4].it is not considered silent and harmless as it is linked with indistinct neurological defect, cognitive impairment, psychological abnormalities, clinically manifest stroke, and early fatality ${ }^{[4]}$. A number of risk conditions are correlated with growing happiness of silent cerebral infarction, the most prominent of which is elevated blood pressure and increasing age ${ }^{[10]}$. Owing to sophisticated advancement of neuroimaging, greater number of patients with atrial fibrillation ascertained to have tiny ischemic brain lesions, that is because of its higher prevalence in individuals with atrial fibrillation and its greater danger to advance to clinically overt neurological defects it should be considered appreciably while physician assess individuals with atrial fibrillation [4]. Investigators perceives that silent infarctions often confined to cerebral cortex and cerebellum are commonly discovered in patients with atrial fibrillation for which cardiac origin is of paramount role ${ }^{[11]}$.

\section{Methodology}

Type of study: this is a case control study.

The study had been long lasting from the beginning of first week of February 2018 to the end of the last week of April 2019.

Study Population: The study population comprises an admitted patient in cardiac care unit in Al-Hussein teaching hospital in Al-Nasiriya city. The diagnosis of cases rely on any accessible tests or reports in case file of patients or diagnosed on interview. Total number of patients that were included in the study was (150), with (50) case with paroxysmal atrial fibrillation, (50) case with persistent atrial fibrillation, and (50) controls.
Inclusion Criteria: Any case with atrial fibrillation with any predisposing factors whether paroxysmal or persistent was included in the study.

\section{Exclusion Criteria:}

1. Any patient with implantable heart device had been inserted or any other contraindication to magnetic resonance imaging.

2. Any patients with existing or antecedent clinical confirmation of transient ischemic attack or cerebrovascular accident.

3. Any recognized case of intracranial injury, space lesions, autoimmune, or inflammatory insults of brain that interfere with precise interpretation of radio imaging.

4. Any patient submitted to trans-catheter procedure for atrial fibrillation.

5. Any case of atrial fibrillation due to valve disorder.

6. Any patient with significant carotid artery stenosis more than seventy percent detected by carotid Doppler sonorographic study.

Sample Size: was convenient restricted by accessibility of patients and time span of study, but sampling task for control, systematized random sampling procedure was done to collect control.

Ethical Issue: An ethical consensus was acquired from Al-Hussein teaching hospital administration. An informed consents also were extracted from all participants.

\section{Study Appliances:}

The Questionnaire: Distinct design of questionnaire was formulated to assemble data and it was revised and reviewed by three subjects matter experts (of community medicine and physician) for examining the validness and the enrichment of questionnaire. The questionnaire is consisted of two sections:

First Section: Involve questions regarding identity information, Second section: questions related to past medical history comprising chronic medical diseases, medication history, type and number of medications have been determined for every individual in the sample (cases and control).

Diagnostic Procedures: Atrial fibrillation is diagnosed by electrocardiogram. "Atrial fibrillation is 
characterized by the replacement of consistent $\mathrm{P}$ wave by rapid oscillation or fibrillatory waves that vary in amplitude, shape, timing associated with an irregular frequently rapid ventricular response when (AV)

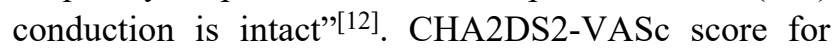
stroke risk estimation in atrial fibrillation was calculated for all individuals suffered from atrial fibrillation [14]. Atrial fibrillation type and time span were delineated. A comprehensive physical examination was carried out for all patients, neurological examination was carried out in agreement with Coma Neurological Check ${ }^{[15]}$. All patients undergone carotid artery Doppler sonographic study to preclude significant carotid artery stenosis greater than seventy percent in agreement with North American symptomatic carotid endarterectomy study ECST, or peak systolic velocity greater than $130 \mathrm{~cm} / \mathrm{s}$ ${ }^{[16]}$. All participants were exposed to magnetic resonance imaging (MRI) study. All magnetic resonant imaging reports were interpreted by two expert radiologist senior make unmindful to clinical informations. The image protocols constitute of sagittal T2 weighted-spin-echo sequence, an axial T2-fluid attenuated inversion recovery (FLAIR) sequence, and a diffusion weighted sequence. Most studies defined silent cerebral ischemia as centerpiece, crisply delineated abnormality greater than three millimeter which is hyperintense on T2 weighted image, a small of number of studies implement ancillary specifications to discriminate silent cerebral infarctions from the quite tiny lesion called leukoaraiosis which is hardly to be categorized as silent infarction, the most pivotal of these specifications is size greater than three millimeters ${ }^{[9]}$. All control individuals with sinus rhythm are gender, age, educational level, and risk factors matched and exposed to preconstructed inquiry sheet of questionnaire comprised of identity informations as for cases, past medical history and chronic medical illnesses including cardiovascular disorders, drug history including antithrombotic. All control subjects are exposed to physical and neurological examination with magnetic resonance imaging. Anatomical locality, laterality and number of lesions on magnetic resonant imaging were predetermined for all cases and controls.

Statistical analysis: statistical package for social sciences (SPSS) version 25 had been used for data assessment, descriptive statistics, frequencies, percentages, associations, tests of significance (chisquare, Fischer exact test, T-test, ANOVA test) had been utilized for interpretation of categorical variables, means, and standard deviation were utilized to present data of continuous variables. A $p$ value less than 0.05 was set as statistically significant.

Results:

A total of 150 individuals engaged with in the study, dispersed evenly in three categories (paroxysmal, persistent atrial fibrillation patients and control group) they exhibit no significant statistical difference in their bio-demographic characters, where $P$ value estimated by ANOVA and chi-square to be greater than 0.05 , in all categories of comparability as expressed in table 1 and 2 .

Table 1: Age parameters of the studied population

\begin{tabular}{|l|c|c|c|c|c|c|}
\hline \multirow{2}{*}{ Age by years } & \multirow{2}{*}{$\mathbf{N}$} & \multirow{2}{*}{ Mean } & \multirow{2}{*}{ Std. Deviation } & \multicolumn{2}{|c|}{$\mathbf{9 5 \% \text { Confidence Interval for Mean }}$} & \multirow{2}{*}{$\begin{array}{c}\text { ANOVA } \\
\text { P value }\end{array}$} \\
\cline { 5 - 6 } & & & & Lower Bound & Upper Bound & \\
\hline Paroxysmal AF & 50 & 66.0200 & 15.27769 & 61.6781 & 70.3619 & 0353 \\
\hline Persistent AF & 50 & 64.4200 & 15.57875 & 59.9926 & 68.8474 & 0.703 \\
\hline Control & 50 & 63.5600 & 13.66832 & 59.6755 & 67.4445 & \\
\hline Total & $\mathbf{1 5 0}$ & $\mathbf{6 4 . 6 6 6 7}$ & $\mathbf{1 4 . 8 0 0 5 4}$ & $\mathbf{6 2 . 2 7 8 7}$ & $\mathbf{6 7 . 0 5 4 6}$ & \\
\hline
\end{tabular}

Anticoagulant * type 


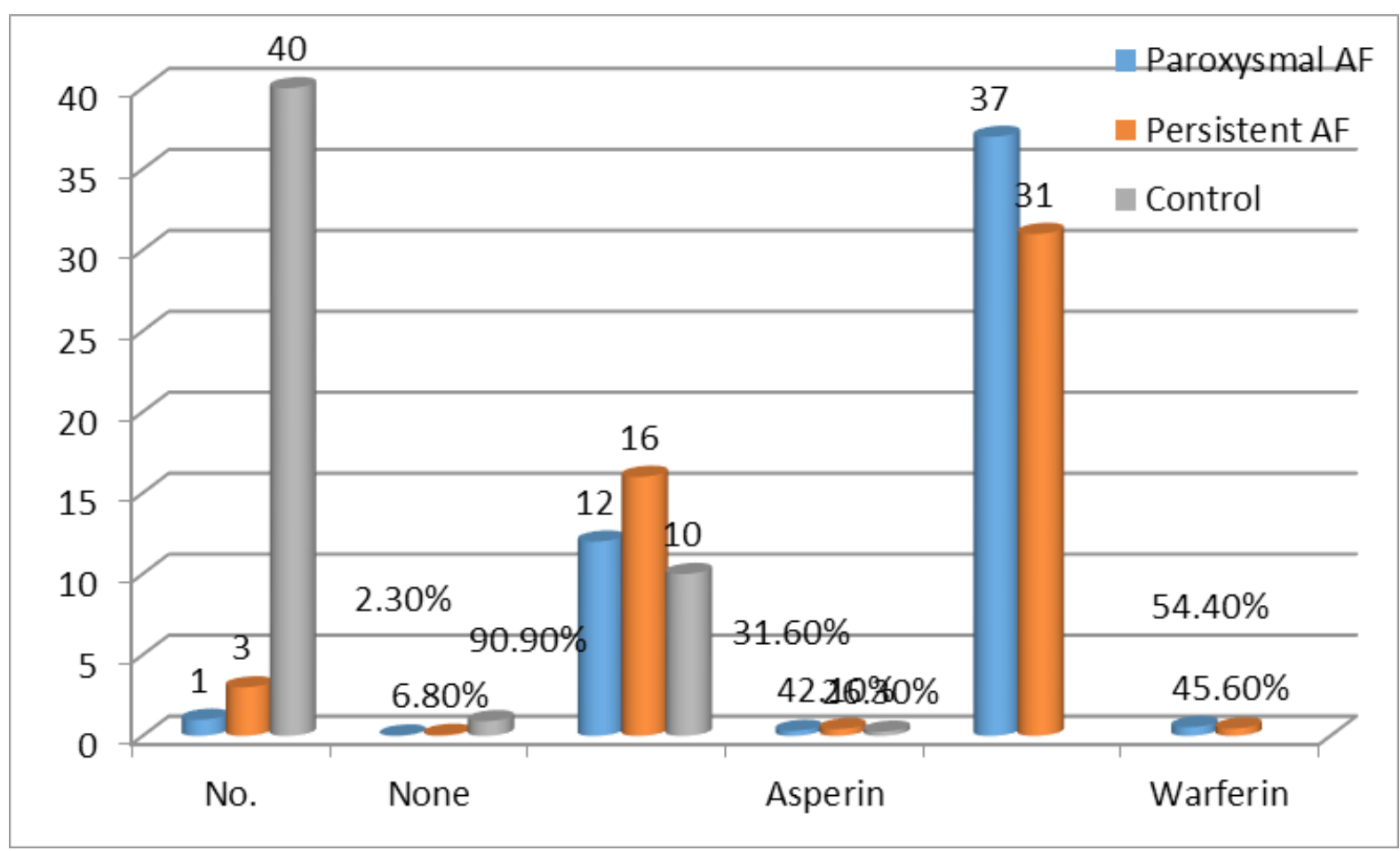

Figure 1: Distribution according to the anticoagulants medications

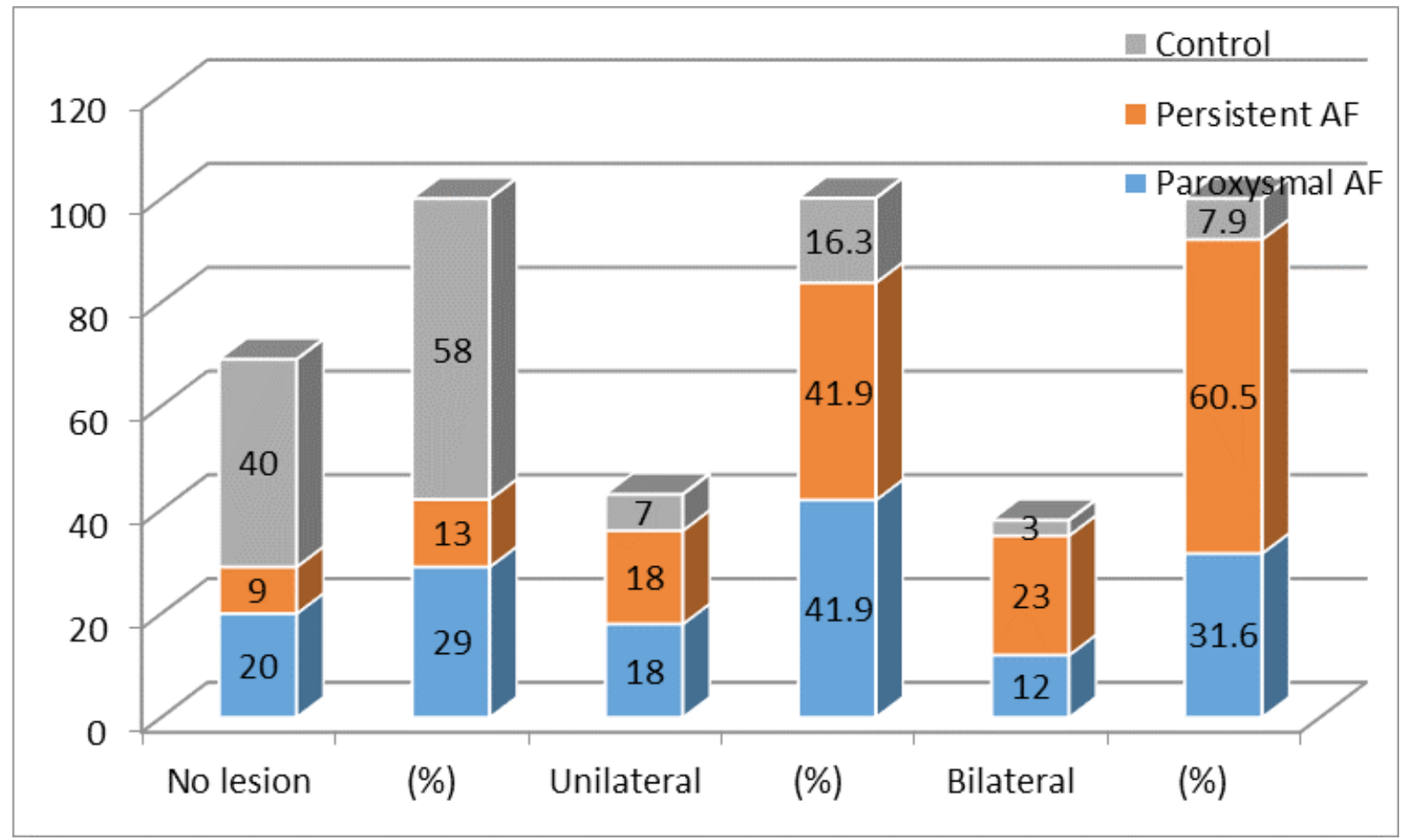

Figure 2: relationship between Laterality with type of studied population

There was significant statistical difference with in the studied group, where Pearson Chi-Square $=42.948^{\mathrm{a}} \mathrm{P}$ value $=0.0001$. As shown in figure 5 . 


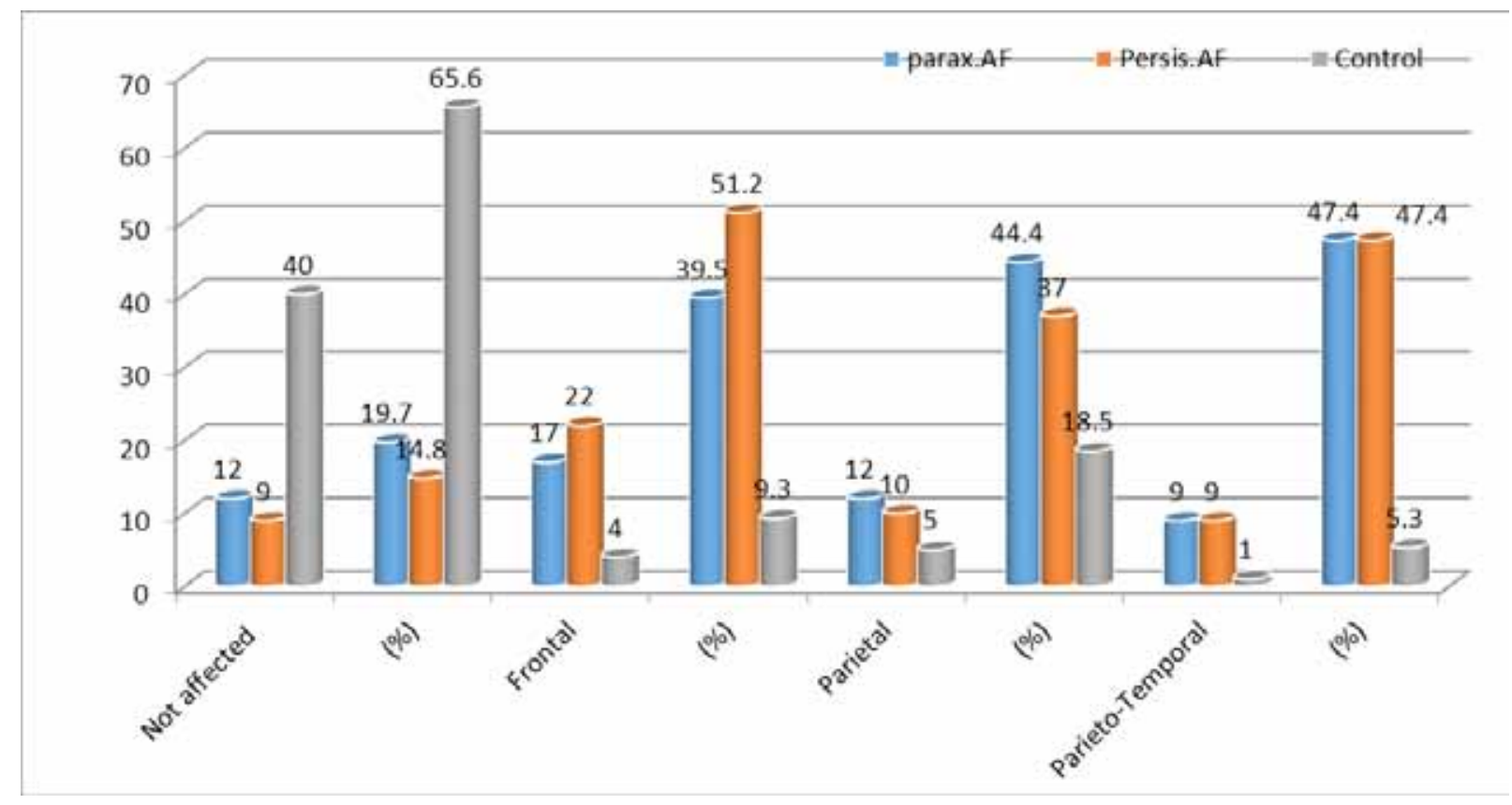

Figure 3: Relationship between locations of stroke with type of studied population

There was significant statistical association between the cases and control and the location of stroke.

Table 2: Distribution of the locations of stroke among studied population

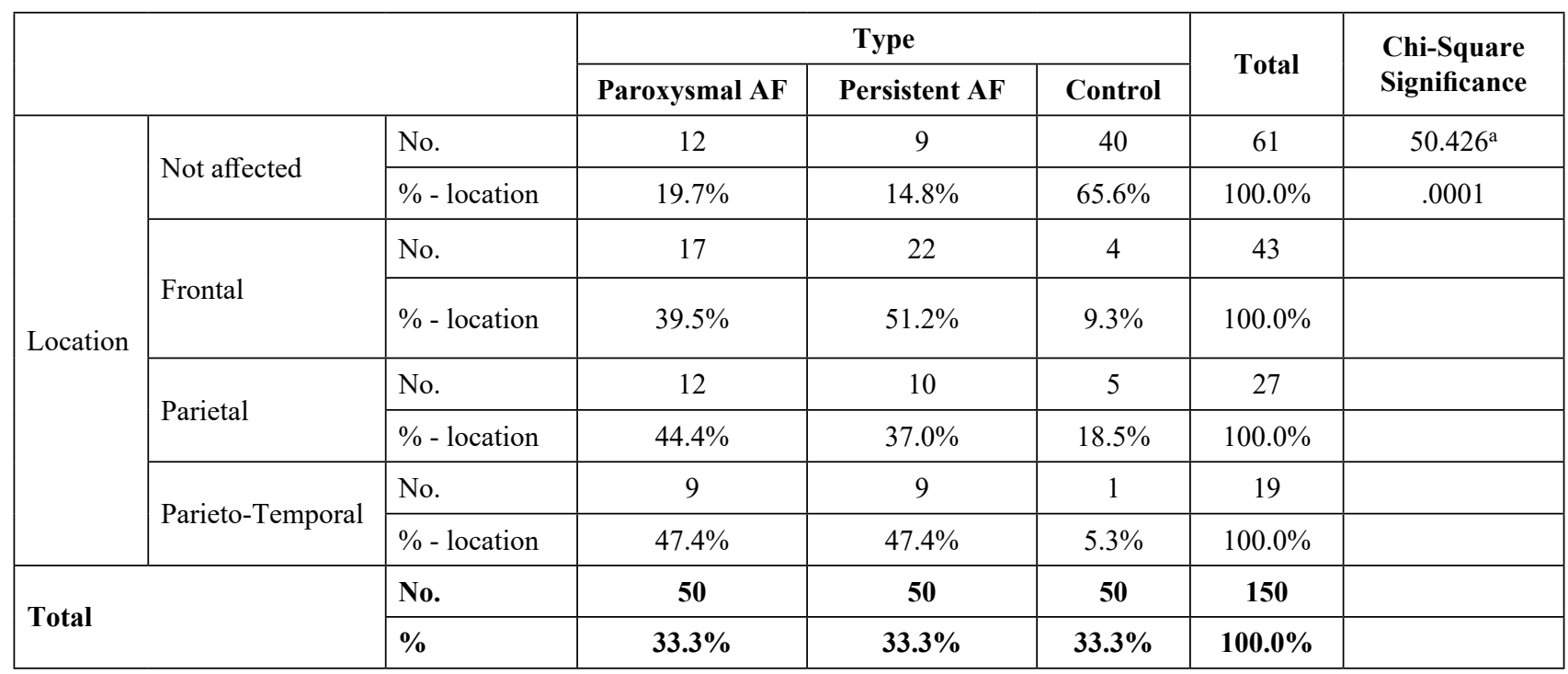

\section{Discussion}

In our study we establish high percentage of silent cerebral ischemia in the analyzed specimen, $59.3 \%$ of all persons engaged in the study were affected by the lesion, $76 \%$ of paroxysmal atrial fibrillation, $82 \%$ of persistent atrial fibrillation while only $20 \%$ of controls were affected by lesions with significant statistical difference between both cases of paroxysmal and persistent atrial fibrillation with control where P value 0.0001. Shadi et $\mathrm{al}^{\left[{ }^{[9]}\right.}$ metaanalysis states that silent cerebral lesions are habitually catch by magnetic resonant imaging in individuals with atrial fibrillation. In current study, Two-sided lesions were observed in $60.5 \%$ of cases with persistent atrial fibrillation while they were observed in $31.6 \%$ of cases with paroxysmal atrial fibrillation. Control subjects have the least percent of two-sided lesions $(3 \%)$. Our findings are in accordance with Fiorenzo et al ${ }^{[18]}$ that demonstrate silent cerebral ischemia is more predominant in cases of atrial fibrillation than in non-atrial fibrillation persons ( $p$ value 0.001 ) and 
persistent atrial fibrillation have had greater percentage of lesions and more bilateral lesions than paroxysmal type. The higher percentage of silent cerebral lesion (46\%) in the control group found by Fiorenzo et al ${ }^{[18]}$ in comparability of $20 \%$ in our study probably linked to difference in the baseline properties of control side in the two studies. But this detection is in accordance with Jonathon et $\mathrm{al}^{[19]}$ a population-obtained studies that displayed prevalence of these lesions of ten to twenty percents. Our study implying that atrial fibrillation is a crucial hazard for silent cerebral ischemia as a cardinal detection, according to Shadi et al ${ }^{[9]}$ atrial fibrillation is accompanied by double fold heighten hazard of silent cerebral infarction. In our study the persistent atrial fibrillation have their lesions located more in frontal lobe bilaterally. This major detection in our study is in line of Fiorenzo et $\mathrm{al}^{[18]}$ study and highlight the method of cardio embolization that is accountable for higher prevalence of silent cerebral infarction in atrial fibrillation which is quite tiny, widely distributed bilaterally principally in frontal lobe ${ }^{[20]}$. Regarding bio demographic characters of the study cases and controls were crossly fit to keep away from selection bias so there was no significant statistical association between cases and controls with bio demographic properties. Though there is significant statistical difference between cases and controls regarding anticoagulant use and Fiorenzo et $\mathrm{al}^{[18]}$ determine identical finding $\mathrm{P}$ value $<0.01$ ), but there is inadequate usage of anticoagulant among cases in our sample, $47 \%$ of paroxysmal atrial fibrillation cases and $44 \%$ of cases with persistent type are mandatory to use anticoagulants according to CHA2DS2VASc score, while only $37 \%$ of paroxysmal and $21 \%$ of persistent atrial fibrillation are undergone anticoagulation, preceding studies point to decreased utilization of warfarin ${ }^{[17]}$.

\section{Conclusion}

Atrial fibrillation is associated with heightened hazard of subclinical cerebral infarction.

Conflict of Interest: None

Funding: Self

Ethical Clearance: Not required

\section{References}

1. Axel B, Marcelle D S,Bao O N,Michiel R,et al.Risk Factor Management In Atrial Fibrillation.
Arrhythmia \& Electrophysiology Review 2018; volume 7, issue (2), pages:118-27: https://doi. org/10.15420/aer.2018.18.2.

2. AnneB.C, Roshan K, Alexander H, et al. Arrhythmia in Patients $>80$ Years of Age: Pathophysiology, Management and Outcomes. Journal of American College of cardiology. 8 May 2018, volume71, issue (18): pages: 2041-2057.https:doi.org/10.1016/j. jacc.2018.03.019.

3. David C. Epidemiology of atrial fibrillation. European Heart Journal, volume 39, issue 16,21 April 2018, pages: 1323-1324.https://doi. org/10.1039.eurheartj/ehy171.

4. Hahne K, Monnig G, Samol A, Atrial fibrillation and silent stroke: links, risks and challenges. Vascular Health and Risk Management.7 March 2016, volume 12, pages:65-74.DOI: https://doi. org/10.2147/VHRM.S81807.

5. Valery L.F, Bo N,George A.et al.Global Burden of Stroke. Circulation Reasearch.3 February,2017;120: 439-448. https://doi. org/10.1161/CIRCRESAHA.116.308413.

6. Narayanaswamy V,Byung W Y,Jeyaraj P,et al.Stroke Epidemiology in South,East,and southEast Asia :A Review. Journal of stroke.2017. sep;19(3): 286-294.doi: 10.5853/jos.2017.00234. PMCID: PMC5647629.

7. Mohamad A MD,Fahad A MD,Sami A MD,etal. Burden Of Atrial Fibrillation-Associated Ischemic Stroke In the United States. JACC:Clinical Electrophysiology, volume 4, Issue 5,2 May 2018, pages :618-625, https://doi.org/10.1016/j. jacep.2018.02.021.

8. Raymond B. F, Michael W R.Identification of Patients at Risk of Stroke from Atrial Fibrillation. US Cardiology Review 2016; volume 10, issue (2); pages:60-4,DOI: https://doi.org/10.15420/ usc. 2016:1:1.

9. Shadi K,Hakan A,Randy L,et al.Association Between Atrial Fibrillation and Silent Cerebral Infarctions: A systemic Review and Metaanalysis. Annals of Internal Medicine. 2014 Nov 4; volume 161.issie(9):pages:650-658. doi:10.7326/M140538.PMCID:PMC5578742.

10. Jonathon P.F, Allan J.W, Andrew A.W, et al. Emerging Spectra of Silent Brain Infarction. Stroke. 7 Oct 2014;45:3461-3471. https://doi.org/10.1161/ STROKEAHA.114.005919. 
11. Keita M,Makoto N,Kentaro A,,et al.Risk factors and localization of silent cerebral infarction in patients with atrial fibrillation. Heart Rhythm .2019 Sep;16(9): 1305-1313.doi: 10.1016/j. hrthm.2019.03. 013.Epub2019 Mar 18.

12. Oreoluwa $\mathrm{O}$ and Ifeanyi N.Stroke risk stratification in atrial fibrillation: A review of common risk factors. Journal of community Hospital Internal Medicine Perspectives.2019 Apr; volume9, issue (2), pages: 113-120.doi: $\quad$ 10.1080/20009666.2019.1593781. PMCID: PMC6484493.PMID:31044042.

13. Hisashi O, MD Yoshimori A, MD Syuhei I,et al.Progression From Paroxysmal to Sustained Atrial Fibrillation Is Associated With Increased Adverse Events.Stroke.5Sep 2018.;49(10):2301-2308.https://doi.org/10.1161/ STROKEAHA.118.021396.

14. Tae-Hoon K, MD pil-Sung Y, MD daehoon K, et al. CHA2DS2-VASc Score for Identifying Truly LowRisk Atrial Fibrillation for Stroke.STROKE.2017; volume 48, pages:2984-2990.https://doi. org/10.1161/STROKEAHA.117.018551.

15. Laura A 1, Celia W, Kathy M.F,et al. Standardized neurological assessments. J Neurosci Nurs.2014 Apr; volume 46, issue (2), pages: 125-132. doi:10.1097/JNN.0000000000000035.

16. Seyman,E.Bornstein,N., Auriel,E.et al.Assessment of carotid artery ultrasonography in the presence of an acoustic shadow artifact.BMC Neurol. 19, 178(2019)https://.doi:10.1186/s12883-019-1405-4. PMCID:PMC6664737.PMID:31357950.

17. Lyn-Hyang L,Hyunah K,Nam K,et al.Underutilization of warfarin for stroke prophylaxis in patients with atrial fibrillation or atrial flutter in Korea. Journal of cardiology.2015 Dec, volume 66, Issue (6), pages: 475-481.doi: 10.1016/j.jjcc.2015.06.013.Epub 2015 Aug 1.

18. Fiorenzo G MD,Lauro C MD,PhD,Matteo A $\mathrm{MD}, \mathrm{PhD}$ et al.Prevalence Of Silent Cerebral Ischemia in Paroxysmal and PersisitentAtrial Fibrillation and Correlation with CognitiveFunction. Journal of the American College of cardiology. Volume 62,Issue 21,pages:1990-1997,10 July 2013.https://doi.org/10.1016/j.jacc.2103.05.47.

19. Jonathon $P$ F Andrew A W,Jhon F F. The epidemiology of silent brain infarction: a systemic review of population-based cohorts.BMC Med.2014;12:119.https://doi.org/10.1186/s12916014-0119-0.PMCID:PMC4226994.

20. Emanuele G,Saverio D,Dario P,et al.Cognitive function and Atrial Fibrillation: from strength of Relationship to Dark side of Prevention. Is there contribution from Sinus Rhythm Restoration and Maintenance. Medicina.2019,volume 55,Issue 9,page:587.https://doi.org/10.3390/medicina 55090 . 